\title{
Neighborhood greenspace and risk of type 2 diabetes in a prospective cohort: the Multi- Ethnic Study of Atherosclerosis
}

\author{
Annie Doubleday ${ }^{1 *+}\left(\mathbb{D}\right.$, Catherine J. Knott ${ }^{2 \dagger}{ }^{\text {, Marnie F. Hazlehurst }}{ }^{2}$, Alain G. Bertoni ${ }^{3}$, Joel D. Kaufman ${ }^{1,2,4}$ and
} Anjum Hajat $^{2}$

\begin{abstract}
Background: Neighborhood greenspaces provide opportunities for increased physical activity and social interaction, and thus may reduce the risk of Type 2 diabetes. However, there is little robust research on greenspace and diabetes. In this study, we examine the longitudinal association between neighborhood greenspace and incident diabetes in the Multi-Ethnic Study of Atherosclerosis.

Methods: A prospective cohort study ( $N=6814 ; 2000-2018)$ was conducted to examine the association between greenspace, measured as annual and high vegetation season median greenness determined by satellite (Normalized Difference Vegetation Index) within $1000 \mathrm{~m}$ of participant homes, and incident diabetes assessed at clinician visits, defined as a fasting glucose level of at least $126 \mathrm{mg} / \mathrm{dL}$, use of insulin or use of hypoglycemic medication, controlling for covariates in stages. Five thousand five hundred seventy-four participants free of prevalent diabetes at baseline were included in our analysis.

Results: Over the study period, 886 (15.9\%) participants developed diabetes. Adjusting for individual characteristics, individual and neighborhood-scale SES, additional neighborhood factors, and diabetes risk factors, we found a $21 \%$ decrease in the risk of developing diabetes per IQR increase in greenspace (HR: 0.79; 95\% Cl: 0.63, 0.99).

Conclusions: Overall, neighborhood greenspace provides a protective influence in the development of diabetes, suggesting that neighborhood-level urban planning that supports access to greenspace--along with healthy behaviors--may aid in diabetes prevention. Additional research is needed to better understand how an area's greenness influences diabetes risk, how to better characterize greenspace exposure and usage, and future studies should focus on robust adjustment for neighborhood-level confounders.
\end{abstract}

Keywords: Neighborhood greenspace, Environmental epidemiology, Type 2 diabetes

\section{Background}

Type 2 diabetes is increasingly common in the U.S. and worldwide, with the age-adjusted prevalence of

${ }^{*}$ Correspondence: doubleda@uw.edu

${ }^{\dagger}$ Annie Doubleday and Catherine J. Knott are co-first authors.

${ }^{1}$ Department of Environmental and Occupational Health Sciences,

University of Washington, Seattle, WA, USA

Full list of author information is available at the end of the article diagnosed type 2 diabetes increasing from $6.4 \%$ in 1990 2002 to $9.4 \%$ in 2013-2016 in the U.S. [1]. Research has shed light on the role of diet, exercise, and adiposity in type 2 diabetes, and prior work has emphasized the importance of individual risk factors [2]. The role of environmental factors, particularly at the neighborhood scale, is less well understood. However, ongoing work indicates that an individual's residential neighborhood may influence an individual's risk [3, 4]. otherwise in a credit line to the material. If material is not included in the article's Creative Commons licence and your intended use is not permitted by statutory regulation or exceeds the permitted use, you will need to obtain permission directly from the copyright holder. To view a copy of this licence, visit http://creativecommons.org/licenses/by/4.0/. The Creative Commons Public Domain Dedication waiver (http://creativecommons.org/publicdomain/zero/1.0/) applies to the data made available in this article, unless otherwise stated in a credit line to the data. 
Increasingly, research has focused on the role of the larger neighborhood social and physical environments, including modifiable community-scale factors such as greenspace, in diabetes risk and prevention. For example, research has explored neighborhood deprivation and other measures of neighborhood socioeconomic status (SES), neighborhood walkability, neighborhood social support and social cohesion, neighborhood safety, and neighborhood greenspace as factors relevant to diabetes [3-6]. In particular, there is a growing body of research on the association between neighborhood greenspace and diabetes [7-16]. Greenspace is often conceptualized as the amount of or proximity to greenness or vegetation, which, in urban settings, includes parks, forests, fields, and outdoor recreation areas [5]. Greenspace is hypothesized to act through multiple mechanisms, including through increasing physical activity and reduced stress. However, the degree to which greenspace operates independently on diabetes is not well understood [3-6].

Most studies examining associations between greenspace and diabetes have used a cross-sectional study design [7-13]. Longitudinal analyses generally suggest an association between cumulative greenspace exposure and risk of diabetes, though there is variability in exposure assessment and results across these studies [14-16]. Cohort studies in the UK and the US identified an inverse relationship between greenspace and risk of diabetes, but are limited by self-reported outcome data or minimal racial and ethnic diversity $[14,15]$. A study using national medical claims data in Taiwan observed this relationship in cross-sectional but not longitudinal analyses, though was limited in adjustment for potential confounders including time-varying and neighborhoodlevel confounders [16]. Our study is poised to fill these gaps by investigating the association between greenspace and incident diabetes in the U.S. in a well characterized cohort with clinician and lab-based diabetes diagnoses and longitudinal covariate data.

In this study, we examine the association between greenspace exposure and the development of incident diabetes in the Multi-Ethnic Study of Atherosclerosis (MESA). This study is the first longitudinal study examining greenspace exposure and incident diabetes in the U.S. with longitudinal covariate data.

\section{Methods}

\section{Study population}

This study was conducted with data from MESA and its ancillary studies. MESA is a prospective cohort assembled initially to understand the relationship between subclinical cardiovascular disease markers and the incidence of cardiovascular disease. At baseline, the MESA cohort comprised 6814 non-institutionalized adults who were cardiovascular disease-free, aged 45-84years, who self-identified as white, black, Hispanic, or Chinese from 6 study sites: New York, NY, Baltimore, MD, Forsyth County, NC, Chicago, IL, St. Paul, MN, and Los Angeles, CA [17]. In this analysis, we further restricted the analytic sample to the 5574 participants who were diabetes free at baseline. The study included follow-up phone questionnaires every 9-12 months. The baseline exam occurred from July 2000 through August 2002, and five follow-up in-person exams occurred an average of $1.6,3.2,4.8,9.5$, and 15.8 years later. The cohort included 5413; 5184; 5080; 4155; and 2962 participants without diabetes at each of the follow-up exams, respectively. Informed consent was obtained from all participants, and the study was approved by the institutional review boards at each study site.

\section{Outcome}

Type 2 diabetes status was ascertained at each followup clinical visit (exam) for study participants, and all participants with prevalent type 2 diabetes at baseline were excluded from this analysis. Using criteria from the American Diabetes Association [18], incident cases were defined as having a fasting glucose level of at least $126 \mathrm{mg} / \mathrm{dL}$ at the exam, use of insulin, or use of hypoglycemic medication. This study considered all incident cases as type 2 diabetes; however, these cases may include patients who developed Latent Autoimmune Diabetes in Adults (LADA). Additionally, because participants were evaluated for diabetes at exam intervals, the diabetes diagnosis dates were subject to interval censoring. Due to the uncertainty of the exact onset of diabetes, we assigned the date of diabetes diagnosis as the midpoint between the prior exam and the exam where the diagnosis was given.

\section{Exposure}

Greenspace was characterized using the Normalized Difference Vegetation Index (NDVI), updated at each exam to match changes in residence. NDVI is a commonly used measure of greenspace derived from satellite imagery [19-21]. For our analysis, NDVI from 2006 satellite imagery with a $250 \mathrm{~m}$ resolution was considered on a scale from 0 to 255-pixel brightness [22]. On this scale, areas with dense vegetation have values around 200, and non-vegetative surfaces such as roads and buildings have values less than 50 .

We obtained participant residence information from the MESA Neighborhood Ancillary study. Home addresses were obtained at baseline and during all follow-up phone calls during the study period. At each participant residence, we characterized greenspace within a varying radius (buffer) to capture greenspace in the 
participant's residential neighborhood. For our primary analysis, we used the annual median NDVI within a $1 \mathrm{~km}$ radius as our a priori exposure, based on prior research indicating that a $1 \mathrm{~km}$ radial buffer had the highest correlation with self-described neighborhood for the corresponding spatial resolution of the NDVI data [21]. NDVI was linked to the address reported at each exam to account for differences in exposure across the study period due to a change in residential address. In sensitivity analyses, we examined annual median NDVI within $500 \mathrm{~m}$ and $2.5 \mathrm{~km}$, as well as high vegetation season (April 1 - September 30) median NDVI within a $1 \mathrm{~km}$ radius. These two time periods were used because NDVI can vary dramatically based on season.

\section{Covariates}

MESA ascertained important potential confounders through a combination of interviews, exams, and census data [17]. We included several individual sociodemographic characteristics: sex, age, race and ethnicity (non-Hispanic white, non-Hispanic black, Hispanic/ Latinx and Chinese), education level (less than or equal to high school, some college, bachelor's degree or higher), employment status (working outside the home or not) and income level. Participant income was calculated from the midpoint of total annual household income specified in categories divided by number of household members $(<\$ 12,000 ; \$ 12,000-24,999 ; \quad \$ 25,000-39,999$, $\$ 40,000-74,999,>\$ 75,000)$. We also included several neighborhood-level characteristics to control for neighborhood-level confounding: neighborhood deprivation index (NDI) [3, 4], social cohesion [23-25], safety [26-28], walkability score $[3,6]$, and proportion urban all measured at the census-tract level. NDI is comprised of several variables from U.S. Census and American Community Survey data representing characteristics of education, occupation and employment, household income and wealth, poverty, and housing, and was assessed at exams 1, 3, and 4 [29-32]. Higher index values indicate greater neighborhood deprivation (i.e., lower neighborhood SES) [32]. Social cohesion, safety, and walkability scores were weighted averages by census tract based on survey responses from MESA participants at baseline and a random sample of people living in the same census tracts as MESA participants in 2004, as in prior MESA research [33]. Social cohesion was measured with a four-item scale including items about willingness to help neighbors, getting along with neighbors, neighborhood trust, and sharing values with neighbors. Safety was measured using a two-item scale about feeling safe in one's neighborhood and violence in the neighborhood. Walkability was measured using a four-item scale about the perceived ease of walking to places in the neighborhood, seeing others walking or exercising, and pleasantness of walking in the neighborhood. Proportion urban measures the percentage of the census tract population living in an urban area, measured using U.S. Census data [30]. Finally, our primary model is additionally adjusted for study site due to the differing population characteristics and NDVI by site.

We additionally adjusted for type 2 diabetes risk factors including family history of diabetes, chronic stress, alcohol consumption, smoking status, body mass index (BMI), and physical activity score. Family history of diabetes was coded dichotomously from responses to a family medical history questionnaire answered at exam 2. Chronic stress was determined from the Chronic Burden scale, a six-item questionnaire given at exams 1 and 3 [34]. Participants were categorized as chronically stressed if they had indicated 6 or more months of strain or difficulties from a relationship, job, or finances, or 6 or more months of medical-related issues for themselves or someone close to them. As in prior MESA research [35], alcohol consumption was categorized as heavy, moderate, and not current drinker and smoking status was categorized as current, former, and never using data available at exams 1-6. BMI was assessed at exams 1-6. Physical activity score was assessed at exams 1-3 and 5, and is the sum of a reverse measure of sedentary behavior, in quartiles $(0=$ most sedentary, $3=$ least sedentary) and a measure of moderate-vigorous physical activity (MVPA), in quartiles $(1=$ least MVPA, $4=$ most MVPA), resulting in a physical activity score from 1 to 7 [36]. Additionally, several of the model covariates varied over each of the 6 exams in order to more accurately capture and control for possible confounding factors. These include: chronic stress, income, employment status, alcohol consumption, smoking status, BMI, physical activity score, and NDI. For all time-varying covariates, data were carried forward if they were missing from future follow-up exams.

\section{Statistical approach}

We conducted a survival analysis examining the association between greenspace exposure and incident diabetes in the MESA cohort. We used Cox proportional hazards regression with time-varying covariates with calendar time as the time scale to estimate the hazard ratio (HR) of incident diabetes risk for an interquartile (IQR) increase in NDVI [35, 37]. Participants were considered in the risk set until diagnosis of diabetes, last follow-up visit, or at the end of the study period (exam 6), whichever occurred first. In a sensitivity analysis, we re-ran the models to estimate the HR for a 0.1 increase in NDVI, on a -1 to 1 scale to compare with results in the literature $[5,38]$.

We determined confounders a priori, and they were added in stages. Model 1 adjusted for age, sex, race/ 
ethnicity, study site, education level, income, employment status, neighborhood deprivation index, neighborhood social cohesion, walkability, safety, and percent urban. The primary model (Model 2) adjusted for the covariates in Model 1 as well as family history of diabetes, BMI, physical activity score, chronic stress, drinking, and smoking. We ran an additional model (Model 3) excluding all potential mediators (BMI, physical activity score, walkability, safety, neighborhood social cohesion) to assess any suggestion of mediation by these factors.

We hypothesized a priori that the association between NDVI and incident diabetes may vary importantly by site, based on the skewed nature of NDVI by site, varying urbanicity, and differing population characteristics in each site. To assess the adequacy of the adjustment for study site using a fixed effect, we conducted a sensitivity analysis using a stratified Cox model with stratification by MESA study site. A stratified Cox model allows the baseline hazard to differ by MESA study site.

We evaluated the assumption of proportional hazards by plotting and testing Schoenfeld residuals and assessing the trend over time. In sensitivity analyses, we examined additional NDVI exposures $(500 \mathrm{~m}$ and $2.5 \mathrm{~km}$ buffers, and high vegetation season NDVI), and effect modification by urbanicity and by neighborhood deprivation index based on prior work showing evidence that the association between greenspace and diabetes is different for urban versus rural areas [14], and by measures of neighborhood socioeconomic status $[7,12,14]$. We also examined effect modification by sex to compare results with prior studies $[11,16]$.

\section{Results}

Of the 5574 MESA study participants with no prevalent diabetes at baseline, 886 (15.9\%) developed incident diabetes over the study period. Those developing incident diabetes were more likely to be Hispanic or Non-Hispanic Black, more likely to have a high school or GED degree or less, more likely to have lower incomes, and more likely to have a family history of diabetes than those who did not develop diabetes during the study period (Table 1).

The distribution of NDVI is skewed by study site, with some sites having predominantly high NDVI and thus more vegetated landscapes, while others have a wider range of NDVI (Fig. S1). Additionally, the study-level IQR (55) is much larger than each of the site-specific IQRs (ranged between 10.75 and 44), indicating that the range of NDVI at each site is much smaller than in the study overall (Table S1).

Results from the primary Cox proportional hazards regression model (Model 2) indicate an inverse association between greenspace and incident diabetes for both
$1 \mathrm{~km}$ annual (HR: 0.79 (95\% CI: 0.63, 0.99)) and $1 \mathrm{~km}$ high vegetation season median NDVI (HR: 0.74 (95\% CI: $0.57,0.95)$ ). For each IQR increase in NDVI, the risk of developing diabetes is $21 \%$ less among those with higher neighborhood NDVI compared to lower, controlling for individual characteristics, neighborhood-level covariates, and diabetes risk factors. In models with $500 \mathrm{~m}$ and $2.5 \mathrm{~km}$ NDVI buffers, we also find an inverse effect but confidence intervals include the null (Table 2). In models excluding potential mediators (Model 3), we find attenuated hazard ratios for all buffer radii (Table 2). When stratified by site using a stratified Cox model rather than adjusting for site with a fixed effect, the results are nearly identical (Table 3).

In our model adjusted for individual and neighborhood characteristics with a $1 \mathrm{~km}$ annual NDVI buffer (Model 1 ), we find an inverse association between greenspace and incident diabetes, however, confidence intervals include the null (HR: 0.83; 95\% CI: 0.66, 1.03) (Table 2). In models with differing buffers (Table 2), excluding potential mediators (Table 2), and with a stratified Cox model (Table 3 ), the results remain similar.

An analysis examining effect modification by NDI, a measure of neighborhood SES, found no evidence that the association between greenspace exposure and incident diabetes varies by NDI tertile, calculated across the entire study sample (Table 4). Furthermore, we find evidence of effect modification by sex, with female participants experiencing a more protective effect compared to male participants (Table 4). Additionally, we were unable to formally evaluate effect modification by urbanicity due to the highly skewed nature of our data, with nearly all participants living in urban areas.

In a sensitivity analysis evaluating the risk of diabetes per 0.1 increase in NDVI, we find similar but attenuated hazard ratios. In our primary model (Model 2), for each 0.1 increase in NDVI, the risk of developing diabetes is $5 \%$ less among those with higher neighborhood NDVI compared to lower (HR: 0.95; (95\% CI: 0.91, 0.99)) (Table S2).

\section{Discussion}

Overall, we find a significant inverse association between greenspace and incident diabetes in the MESA cohort in our fully-adjusted model (Model 2). Additionally, we observe an attenuated inverse effect with a more parsimonious model (Model 1) and when using a $500 \mathrm{~m}$ or $2.5 \mathrm{~km}$ buffer radius for NDVI exposure, with confidence intervals that include the null. Our results hold when we use a stratified Cox model for study site (Table 3), and when we use an NDVI scale commonly used in the literature (Table 2). We find evidence of effect modification by sex, and no evidence of effect modification by 
Table 1 Participant characteristics at baseline by diabetes status

\begin{tabular}{|c|c|c|c|}
\hline & $\begin{array}{l}\text { No incident diabetes } \\
(N=4688)\end{array}$ & $\begin{array}{l}\text { Incident diabetes } \\
(N=886)\end{array}$ & $\begin{array}{l}\text { Overall } \\
(N=5574)\end{array}$ \\
\hline \multicolumn{4}{|l|}{ Sex } \\
\hline Female & $2530(54.0 \%)$ & 445 (50.2\%) & $2975(53.4 \%)$ \\
\hline Male & $2158(46.0 \%)$ & $441(49.8 \%)$ & $2599(46.6 \%)$ \\
\hline \multicolumn{4}{|l|}{ Age } \\
\hline 45-54 & $1382(29.5 \%)$ & $314(35.4 \%)$ & $1696(30.4 \%)$ \\
\hline $55-64$ & $1265(27.0 \%)$ & $273(30.8 \%)$ & $1538(27.6 \%)$ \\
\hline $65-74$ & 1357 (28.9\%) & $228(25.7 \%)$ & $1585(28.4 \%)$ \\
\hline $75-84$ & $679(14.5 \%)$ & $70(7.9 \%)$ & $749(13.4 \%)$ \\
\hline Missing & $5(0.1 \%)$ & $1(0.1 \%)$ & $6(0.1 \%)$ \\
\hline \multicolumn{4}{|l|}{ Race/Ethnicity } \\
\hline Hispanic & 905 (19.3\%) & $242(27.3 \%)$ & $1147(20.6 \%)$ \\
\hline Non-Hispanic Black & $1182(25.2 \%)$ & $256(28.9 \%)$ & $1438(25.8 \%)$ \\
\hline Non-Hispanic Chinese & $530(11.3 \%)$ & $114(12.9 \%)$ & $644(11.6 \%)$ \\
\hline Non-Hispanic White & $2071(44.2 \%)$ & $274(30.9 \%)$ & $2345(42.1 \%)$ \\
\hline \multicolumn{4}{|l|}{ Education } \\
\hline HS/GED or less & 1535 (32.7\%) & $325(36.7 \%)$ & $1860(33.4 \%)$ \\
\hline Some college & $1318(28.1 \%)$ & $277(31.3 \%)$ & $1595(28.6 \%)$ \\
\hline Bachelors or higher & $1817(38.8 \%)$ & $283(31.9 \%)$ & $2100(37.7 \%)$ \\
\hline Missing & $18(0.4 \%)$ & $1(0.1 \%)$ & $19(0.3 \%)$ \\
\hline \multicolumn{4}{|l|}{ Income Category } \\
\hline$<\$ 12,000$ & $1192(25.4 \%)$ & $257(29.0 \%)$ & $1449(26.0 \%)$ \\
\hline$\$ 12,000-\$ 24,999$ & $1301(27.8 \%)$ & $263(29.7 \%)$ & $1564(28.1 \%)$ \\
\hline$\$ 25,000-\$ 39,999$ & $926(19.8 \%)$ & $181(20.4 \%)$ & $1107(19.9 \%)$ \\
\hline$\$ 40,000-\$ 74,999$ & $927(19.8 \%)$ & $131(14.8 \%)$ & $1058(19.0 \%)$ \\
\hline$\$ 75,000+$ & $143(3.1 \%)$ & $15(1.7 \%)$ & $158(2.8 \%)$ \\
\hline Missing & $199(4.2 \%)$ & $39(4.4 \%)$ & $238(4.3 \%)$ \\
\hline \multicolumn{4}{|l|}{ Employment } \\
\hline Employed & $2333(49.8 \%)$ & $478(54.0 \%)$ & $2811(50.4 \%)$ \\
\hline Unemployed/retired & 2337 (49.9\%) & $406(45.8 \%)$ & $2743(49.2 \%)$ \\
\hline Missing & $18(0.4 \%)$ & $2(0.2 \%)$ & $20(0.4 \%)$ \\
\hline \multicolumn{4}{|l|}{ Site } \\
\hline Forsyth County, NC & $772(16.5 \%)$ & $137(15.5 \%)$ & 909 (16.3\%) \\
\hline New York City, NY & 724 (15.4\%) & $174(19.6 \%)$ & $898(16.1 \%)$ \\
\hline Baltimore, MD & $725(15.5 \%)$ & $130(14.7 \%)$ & 855 (15.3\%) \\
\hline St. Paul, MN & $746(15.9 \%)$ & $136(15.3 \%)$ & $882(15.8 \%)$ \\
\hline Chicago, IL & $881(18.8 \%)$ & $127(14.3 \%)$ & $1008(18.1 \%)$ \\
\hline Los Angeles, CA & 840 (17.9\%) & $182(20.5 \%)$ & $1022(18.3 \%)$ \\
\hline \multicolumn{4}{|l|}{ Family history of diabetes } \\
\hline Family history & $1384(29.5 \%)$ & $388(43.8 \%)$ & $1772(31.8 \%)$ \\
\hline No family history & $3119(66.5 \%)$ & 479 (54.1\%) & $3598(64.5 \%)$ \\
\hline Missing & $185(3.9 \%)$ & $19(2.1 \%)$ & $204(3.7 \%)$ \\
\hline \multicolumn{4}{|l|}{ Chronically stressed } \\
\hline Chronically stressed & $2815(60.0 \%)$ & $529(59.7 \%)$ & $3344(60.0 \%)$ \\
\hline Not chronically stressed & $1822(38.9 \%)$ & $354(40.0 \%)$ & $2176(39.0 \%)$ \\
\hline Missing & $51(1.1 \%)$ & $3(0.3 \%)$ & $54(1.0 \%)$ \\
\hline \multicolumn{4}{|l|}{ BMI } \\
\hline Mean (SD) & $27.5(5.0)$ & $30.8(5.7)$ & $28.0(5.3)$ \\
\hline \multicolumn{4}{|l|}{ Physical activity level } \\
\hline High & 977 (20.8\%) & $168(19.0 \%)$ & $1145(20.5 \%$ \\
\hline
\end{tabular}


Table 1 (continued)

\begin{tabular}{|c|c|c|c|}
\hline & $\begin{array}{l}\text { No incident diabetes } \\
(N=4688)\end{array}$ & $\begin{array}{l}\text { Incident diabetes } \\
(N=886)\end{array}$ & $\begin{array}{l}\text { Overall } \\
(N=5574)\end{array}$ \\
\hline Medium & 2853 (60.9\%) & $546(61.6 \%)$ & 3399 (61.0\%) \\
\hline Low & $843(18.0 \%)$ & $172(19.4 \%)$ & $1015(18.2 \%)$ \\
\hline Missing & $15(0.3 \%)$ & $0(0 \%)$ & $15(0.3 \%)$ \\
\hline \multicolumn{4}{|l|}{ Alcohol consumption } \\
\hline Heavy & $726(15.5 \%)$ & $122(13.8 \%)$ & $848(15.2 \%)$ \\
\hline Moderate & $2021(43.1 \%)$ & 377 (42.6\%) & $2398(43.0 \%)$ \\
\hline Never/not current & 1909 (40.7\%) & $384(43.3 \%)$ & $2293(41.1 \%)$ \\
\hline Missing & $32(0.7 \%)$ & $3(0.3 \%)$ & $35(0.6 \%)$ \\
\hline \multicolumn{4}{|l|}{ Smoking } \\
\hline Current & $686(14.6 \%)$ & $142(16.0 \%)$ & $828(14.9 \%)$ \\
\hline Former & $1774(37.8 \%)$ & $325(36.7 \%)$ & $2099(37.7 \%)$ \\
\hline Never & $2210(47.1 \%)$ & $418(47.2 \%)$ & $2628(47.1 \%)$ \\
\hline Missing & $18(0.4 \%)$ & $1(0.1 \%)$ & $19(0.3 \%)$ \\
\hline \multicolumn{4}{|c|}{ Neighborhood Deprivation Index (NDI) tertile } \\
\hline High SES (low NDI) & $1543(32.9 \%)$ & $253(28.6 \%)$ & $1796(32.2 \%)$ \\
\hline Mid SES (mid NDI) & $1497(31.9 \%)$ & $298(33.6 \%)$ & $1795(32.2 \%)$ \\
\hline Low SES (high NDI) & $1470(31.4 \%)$ & $323(36.5 \%)$ & $1793(32.2 \%)$ \\
\hline Missing & $178(3.8 \%)$ & $12(1.4 \%)$ & $190(3.4 \%)$ \\
\hline \multicolumn{4}{|c|}{ Neighborhood Social Cohesion } \\
\hline High & $1503(32.1 \%)$ & $283(31.9 \%)$ & $1786(32.0 \%)$ \\
\hline Mid & $1510(32.2 \%)$ & $280(31.6 \%)$ & $1790(32.1 \%)$ \\
\hline Low & $1486(31.7 \%)$ & $305(34.4 \%)$ & $1791(32.1 \%)$ \\
\hline Missing & $189(4.0 \%)$ & $18(2.0 \%)$ & $207(3.7 \%)$ \\
\hline \multicolumn{4}{|l|}{ Neighborhood Walkability } \\
\hline High & $1532(32.7 \%)$ & $240(27.1 \%)$ & $1772(31.8 \%)$ \\
\hline Mid & $1478(31.5 \%)$ & $298(33.6 \%)$ & $1776(31.9 \%)$ \\
\hline Low & $1446(30.8 \%)$ & $330(37.2 \%)$ & $1776(31.9 \%)$ \\
\hline Missing & $232(4.9 \%)$ & $18(2.0 \%)$ & $250(4.5 \%)$ \\
\hline \multicolumn{4}{|l|}{ Neighborhood Safety } \\
\hline High & 1505 (32.1\%) & $267(30.1 \%)$ & $1772(31.8 \%)$ \\
\hline Mid & $1490(31.8 \%)$ & $286(32.3 \%)$ & 1776 (31.9\%) \\
\hline Low & $1461(31.2 \%)$ & $315(35.6 \%)$ & $1776(31.9 \%)$ \\
\hline Missing & $232(4.9 \%)$ & $18(2.0 \%)$ & $250(4.5 \%)$ \\
\hline \multicolumn{4}{|l|}{ NDVI (Mean (SD)) } \\
\hline Annual median 1000 m & $127(33.3)$ & $129(33.5)$ & $127.5(33.3)$ \\
\hline
\end{tabular}

Note: All participants are diabetes free at baseline. All characteristics are based on data collected at baseline

neighborhood deprivation (Table 4). Although not the main objective of the paper, we find limited evidence of mediation by a combined set of mediators when using an exploratory mediation analysis approach.

Our results agree with the main findings in the literature that generally report inverse associations between greenspace and diabetes [7-16]. However, it is challenging to compare results across studies examining the association between greenspace and diabetes due to the varying measures of greenspace (proportion greenspace or park space within a buffer, distance to parks, NDVI), differing spatial scales, differing study designs, and vastly different study populations $[5,6$, 20, 21, 26, 38, 39]. Three studies have reported results on the longitudinal association between greenspace and incident diabetes. Dalton et al. found a significant inverse association, with greenspace measured as proportion greenspace within an $800 \mathrm{~m}$ buffer determined from the UK Land Cover Map, comparing the highest to the lowest quartile, and incident diabetes ascertained through self-report (HR: 0.81; 95\% CI: 0.67, 0.99). This study was based in the UK, with a larger cohort than 
Table 2 Hazard ratios (HRs) and 95\% confidence intervals (Cls) for incident diabetes corresponding to an interquartile range (IQR) increase in NDVI

\begin{tabular}{|c|c|}
\hline Buffer size & $\mathrm{HR}(95 \% \mathrm{Cl})$ \\
\hline Model $1^{\mathrm{a}}$ & $0.83(0.66,1.03)$ \\
\hline Model $2^{b}$ & $0.79(0.63,0.99)^{*}$ \\
\hline Model $3^{c}$ & $0.88(0.72,1.08)$ \\
\hline \multicolumn{2}{|c|}{$1 \mathrm{~km}$ radius - high vegetation season median } \\
\hline Model $1^{a}$ & $0.79(0.61,1.01)$ \\
\hline Model $2^{b}$ & $0.74(0.57,0.95)^{*}$ \\
\hline Model $3^{c}$ & $0.84(0.67,1.06)$ \\
\hline \multicolumn{2}{|c|}{$500 \mathrm{~m}$ radius - annual median } \\
\hline Model $1^{\mathrm{a}}$ & $0.86(0.69,1.07)$ \\
\hline Model $2^{b}$ & $0.84(0.67,1.05)$ \\
\hline Model $3^{c}$ & $0.90(0.73,1.09)$ \\
\hline \multicolumn{2}{|c|}{$2.5 \mathrm{~km}$ radius - annual median } \\
\hline Model $1^{\text {a }}$ & $0.90(0.7,1.15)$ \\
\hline Model $2^{b}$ & $0.84(0.65,1.08)$ \\
\hline Model $3^{c}$ & $0.95(0.76,1.20)$ \\
\hline
\end{tabular}

a Model 1 adjustment set: age, sex, race/ethnicity, education category, income category, employment status, neighborhood deprivation index, neighborhood social cohesion, neighborhood walkability, neighborhood safety, urbanicity, and site

${ }^{b}$ Model 2 (primary model) adjustment set: Model 1 adjustment set plus family history of diabetes, BMI, physical activity score, chronic stress, smoking, drinking ' Model 3 adjustment set: site, age, sex race/ethnicity, education category, income category, employment status, neighborhood deprivation index, family history of diabetes, chronic stress, smoking, drinking

* $p \leq 0.05$

Table 3 Hazard ratios (HRs) and 95\% confidence intervals (Cls) for incident diabetes corresponding to an interquartile range (IQR) increase in NDVI from stratified Cox models, stratified by study site

\begin{tabular}{|c|c|}
\hline Buffer size & $\mathrm{HR}(95 \% \mathrm{Cl})$ \\
\hline \multicolumn{2}{|c|}{$1 \mathrm{~km}$ radius - annual median } \\
\hline Model $1^{\mathrm{a}}$ & $0.83(0.66,1.03)$ \\
\hline Model $2^{b}$ & $0.79(0.63,0.99)^{*}$ \\
\hline \multicolumn{2}{|c|}{$1 \mathrm{~km}$ radius - high vegetation season median } \\
\hline Model $1^{\mathrm{a}}$ & $0.79(0.61,1.01)$ \\
\hline Model $2^{b}$ & $0.74(0.57,0.95)^{*}$ \\
\hline \multicolumn{2}{|c|}{$500 \mathrm{~m}$ radius - annual median } \\
\hline Model $1^{\mathrm{a}}$ & $0.86(0.69,1.06)$ \\
\hline Model $2^{b}$ & $0.84(0.67,1.05)$ \\
\hline \multicolumn{2}{|c|}{$2.5 \mathrm{~km}$ radius - annual median } \\
\hline Model $1^{\mathrm{a}}$ & $0.89(0.70,1.14)$ \\
\hline Model $2^{b}$ & $0.84(0.65,1.08)$ \\
\hline
\end{tabular}

a Model 1 adjustment set: age, sex, race/ethnicity, education category, income category, employment status, neighborhood deprivation index, neighborhood social cohesion, neighborhood walkability, neighborhood safety, urbanicity, and site

b Model 2 (primary model) adjustment set: Model 1 adjustment set plus family history of diabetes, BMI, physical activity score, chronic stress, smoking, drinking * $p \leq 0.05$
Table 4 Results from multiplicative interaction by levels of neighborhood deprivation index (NDI) and by sex for the primary model (Model 2)

\begin{tabular}{|c|c|c|}
\hline Model & $1 \mathrm{~km}$ annual median NDVI & $\begin{array}{l}1 \mathrm{~km} \text { high vegetation } \\
\text { season median NDVI }\end{array}$ \\
\hline $\begin{array}{l}\text { Model } 2 \text {, plus } \\
\text { interaction by } \\
\mathrm{NDI}^{\mathrm{a}} \text { ( } p \text {-value) }\end{array}$ & 0.64 & 0.58 \\
\hline $\begin{array}{l}\text { Model 2, plus } \\
\text { interaction by } \\
\text { sex }^{\text {b }} \text { (HR ( } 95 \% \\
\text { CI)) }\end{array}$ & $1.33(1.05,1.69)^{*}$ & $1.39(1.08,1.80)^{*}$ \\
\hline
\end{tabular}

a Reported $p$-values for NDI are derived from an ANOVA comparing the interaction model to a model without an interaction

b Reported coefficients for sex are derived from the interaction term of NDVI and sex, showing the effect of greenspace by sex. Females are the referent category

Adjustment set includes all Model 2 covariates plus interaction term between greenspace and NDI tertile or between greenspace and sex

${ }^{*} p \leq 0.05$

MESA (>23,000 participants), and a similar set of covariates [14]. Further, Lee et al. found a significant cross-sectional association between greenspace, as proportion greenspace by census block group comparing highest to lowest quartile, and prevalent diabetes, defined by fasting plasma glucose $\geq 126 \mathrm{mg} / \mathrm{dL}$, but this association was null in their longitudinal analysis (OR: 0.70; 95\% CI: 0.41, 1.19). Participants in this study were from the a cohort in Massachusetts, with a comparable sized cohort to MESA ( 4000 participants), and similar covariate adjustment [15]. Finally, Tsai et al. found a significant inverse association, with greenspace reported per IQR increase in NDVI among a sample of individuals from Taiwan's National Health Insurance medical claims data (HR: 0.81; 95\% CI: 0.79-0.82). This cohort was substantially larger than MESA $(>400,000)$, with a more limited adjustment set due to the lack of neighborhood-level covariate data [16].

Several other studies have examined the cross-sectional association between measures of greenspace and prevalent diabetes [7-13]. Many found significant inverse associations, however, in some studies, the strength of association was very small [10], or found in only one of several measures of greenspace [8]. Furthermore, among these cross-sectional studies, only two used NDVI as the measure of greenspace, and both reported per 0.1 unit increase in NDVI (on a -1 to 1 scale), with a $500 \mathrm{~m}$ buffer [13] or at the block-level [12]. Moreover, only three studies identified diabetes through blood samples $[9,13,15]$, while four used diagnosis codes or claims data $[8,10,12$, 16], and three relied on self-reported diagnosis [7, 11, 14]. Thus, it is challenging to directly compare our results and to put our results into broader context. 
Our results indicate possible confounding by neighborhood-level factors, including by measures of neighborhood SES. Several studies provide evidence of differential exposure to greenspace by neighborhood SES [3-5, 40]. In particular, individual neighborhood selection is a common methodological issue, and if not adequately controlled for, can lead to biased estimates [3, 4, 41, 42]. In a study where individuals were sampled from the MESA study site locations, authors reported individuals had different residential preferences based on their SES and individual characteristics, indicating that residential self-selection is a critical confounder [42]. In an effort to address this, we adjusted for neighborhood-level proxy measures (social cohesion, safety, walkability) that may account for some of the residual neighborhood-level confounding and residential self-selection not accounted for by adjustment for neighborhood deprivation index. Future studies should collect and adjust for individual residential preferences in order to account for this important source of bias.

The current literature indicates several possible mechanisms through which greenspace may reduce the risk of diabetes $[3,5,6,26,38,39]$. One proposed mechanism suggests that access to greenspace increases physical activity, particularly in urban environments, leading to lower BMI and adiposity, which are associated with lower rates of several chronic conditions, including diabetes [3-5]. However, existing evidence for mediation by measures of physical activity and adiposity is mixed [13, 14]. Other proposed mechanisms include reduced stress, increased social cohesion, and reduction in air and noise pollution, and have not been fully explored for diabetes $[3,5,6$, 13, 23]. Results from Model 3 in this analysis suggest possible mediation by a combination of neighborhood (social cohesion, safety, walkability) and individual (BMI, physical activity) factors. Further mediation analyses are needed to increase the understanding of possible mechanisms. However, methods for mediation analysis with survival outcomes and time-varying mediators need further development [43].

The primary strength of this study is that, in leveraging the MESA cohort, we are able to take advantage of the study's rich longitudinal covariate data, allowing for several time-varying covariates, which are not present in the three other longitudinal studies on greenspace exposure and incident diabetes [14-16]. Furthermore, this study used blood measurements from in-person clinic visits and medication data to ascertain diabetes diagnoses, while previous studies examining greenspace and diabetes often rely on self-reported diabetes diagnosis [7, 11, 14] or administrative data $[8,10,12,16]$. Additionally, this cohort is both racially and ethnically diverse, and represents several regions in the U.S. We also examined the association using different exposure buffers to understand how the association may vary with different neighborhood sizes [21]. Finally, we included several neighborhood-level covariates in an attempt to better control for neighborhood SES and neighborhood-level confounding; many of these covariates are not typically included in other studies [7-16].

There are several limitations of this work that can be improved upon in future research. NDVI is not a specific measure of greenspace and has no information about the quality of greenspaces. While NDVI is a standard measure of greenspace, a higher spatial and temporal resolution of NDVI satellite data sources, or alternate assessments of greenspace exposure may aid in reducing exposure misclassification [3, 20, 21]. Further, while this study has a wealth of longitudinal covariate data, there is no data regarding greenspace usage. Future work should consider alternate definitions of greenspace and take into account greenspace usage and accessibility rather than solely neighborhood vegetation. Additionally, we did not formally account for interval censoring, which may bias our results.

\section{Conclusions}

Overall, we find a significant inverse association from our fully-adjusted model between greenspace exposure and incident diabetes and a non-significant inverse effect found in a more parsimonious model and when using differing neighborhood buffers. We additionally find evidence of moderation by sex, but no evidence of moderation by neighborhood deprivation index. These results indicate, as other studies have found, that the relationship between greenspace and diabetes is complex, and is likely confounded by several individual and neighborhood-level factors. Further work should consider alternate measures of greenspace that take into account greenspace usage, as well as more robust data collection and adjustment for individual and neighborhood SES, individual neighborhood preferences, and other neighborhood-level covariates. This study contributes to the literature suggesting that neighborhood greenspace is modestly protective against diabetes [7-16], indicating that neighborhood-level planning that supports healthy behaviors and lifestyles may aid in diabetes prevention. 


\section{Supplementary Information}

The online version contains supplementary material available at https://doi. org/10.1186/s12940-021-00824-w.

\section{Additional file 1.}

\section{Acknowledgments}

The authors thank other MESA staff, investigators, and participants for their contributions.

\section{Authors' contributions}

Annie Doubleday: Methodology, Software, Validation, Formal analysis, Investigation, Writing - Original Draft, Writing - Review \& Editing, Visualization; Catherine Knott: Conceptualization, Methodology, Software, Validation, Formal analysis, Investigation, Writing - Original Draft, Writing - Review \& Editing; Marnie F. Hazlehurst: Writing - Review \& Editing; Alain Bertoni: Writing - Review \& Editing; Joel D. Kaufman: Methodology, Investigation, Supervision, Writing - Review \& Editing; and Anjum Hajat: Conceptualization, Methodology, Investigation, Supervision, Writing - Review \& Editing. The authors read and approved the final manuscript.

\section{Funding}

This research was supported by contracts 75N92020D00001, HHSN2682015000031, N01-HC-95159, 75N92020D00005, N01-HC-95160, 75N92020D00002, N01-HC-95161, 75N92020D00003, N01-HC-95162, 75N92020D000006, N01-HC-95163, 75N92020D00004, N01-HC-95164, 75N92020D00007, N01-HC-95165, N01-HC-95166, N01-HC-95167, N01-HC-95168 and N01-HC-95169 from the National Heart, Lung, and Blood Institute, and by grants UL1-TR-000040, UL1-TR-001079, and UL1-TR-001420 from the National Center for Advancing Translational Sciences (NCATS). The authors thank the other investigators, the staff, and the participants of the MESA study for their valuable contributions. A full list of participating MESA investigators and institutions can be found at http://www. mesa-nhlbi.org

The data from MESA Air was developed under STAR research assistance agreements, No. RD831697 (MESA Air) and RD-83830001 (MESA Air Next Stage), awarded by the U.S Environmental Protection Agency. It has not been formally reviewed by the EPA. The views expressed in this document are solely those of the authors and the EPA does not endorse any products or commercial services mentioned in this publication. Data from the MESA Neighborhood Study was supported by grant R01 HL071759.

This work was also funded by BEBTEH: Biostatistics, Epidemiologic \& Bioinformatic Training in Environmental Health (NIEHS, T32ES015459), and the University of Washington Center for Exposures, Diseases, Genomics, and Environment (NIEHS, P30ES007033).

\section{Availability of data and materials}

The datasets analyzed during this study are not publicly available due to MESA study requirements.

\section{Declarations}

\section{Ethics approval and consent to participate}

MESA was approved by the Institutional Review Boards of each site, and all participants gave written informed consent.

\section{Consent for publication}

N/A

\section{Competing interests}

Authors declare no conflicts of interest.

\section{Author details}

'Department of Environmental and Occupational Health Sciences, University of Washington, Seattle, WA, USA. ${ }^{2}$ Department of Epidemiology, University of Washington, Seattle, WA, USA. ${ }^{3}$ Department of Epidemiology and Prevention, Wake Forest School of Medicine, Winston-Salem, NC, USA. ${ }^{4}$ Department of Medicine, University of Washington, Seattle, WA, USA.
Received: 18 June 2021 Accepted: 22 December 2021

Published online: 16 January 2022

\section{References}

1. DHHS. National Diabetes Statistics Report, 2020. Natl Diabetes Stat Rep. 2020;2:1-30.

2. National Institute of Diabetes and Digestive and Kidney Diseases. Risk Factors for Type 2 Diabetes [Internet] 2021. Available from: https://www. niddk.nih.gov/health-information/diabetes/overview/risk-factors-type-2diabetes

3. Dendup T, Feng $X$, Clingan S, Astell-Burt T. Environmental risk factors for developing type 2 diabetes mellitus: a systematic review. Int J Environ Res Public Health. 2018;15(1):78. https://doi.org/10.3390/ijerph15010078.

4. Bilal U, Auchincloss AH, Diez-roux AV. Neighborhood environments and diabetes risk and control. Curr Diab Rep. 2018;18(62). https://doi.org/10. 1007/s11892-018-1032-2.

5. De la Fuente F, Saldías MA, Cubillos C, Mery G, Carvajal D, Bowen M, et al. Green space exposure association with type 2 diabetes mellitus, physical activity, and obesity: a systematic review. Int J Environ Res Public Health. 2021;18(1):1-18.

6. DenBraver NR, Lakerveld J, Rutters F, Schoonmade L, Brug J, Beulens JWJ. Built environmental characteristics and diabetes: a systematic review and meta-analysis. BMC Med. 2018;16;12. https://doi.org/10.1186/ s12916-017-0997-z.

7. Astell-Burt T, Feng X, Kolt GS. Is neighborhood green space associated with a lower risk of type 2 diabetes evidence from 267,072 australians. Diabetes Care. 2014;37(1):197-201.

8. Ngom R, Gosselin P, Blais C, Rochette L. Type and proximity of green spaces are important for preventing cardiovascular morbidity and diabetes - a cross-sectional study for Quebec, Canada. Int J Environ Res Public Health. 2016;13:423. https://doi.org/10.3390/ijerph13040423.

9. Bodicoat DH, O'Donovan G, Dalton AM, Gray L, Yates T, Edwardson C, et al. The association between neighbourhood greenspace and type 2 diabetes in a large cross-sectional study. BMJ Open. 2014;4(12):1-8.

10. Maas J, Verheij RA, De Vries S, Spreeuwenberg P, Schellevis FG, Groenewegen PP. Morbidity is related to a green living environment. J Epidemiol Community Health. 2009;63(12):967-73.

11. Müller G, Harhoff R, Rahe C, Berger K. Inner-city green space and its association with body mass index and prevalent type 2 diabetes: a crosssectional study in an urban German city. BMJ Open. 2018:8(1):1-9.

12. Brown SC, Lombard J, Wang K, Byrne MM, Toro M, Plater-Zyberk E, et al. Neighborhood greenness and chronic health conditions in medicare beneficiaries. Am J Prev Med. 2016;51(1):78-89 Available from: https:// doi.org/10.1016/j.amepre.2016.02.008.

13. Yang BY, Markevych I, Heinrich J, Bowatte G, Bloom MS, Guo Y, et al. Associations of greenness with diabetes mellitus and glucose-homeostasis markers: the 33 communities Chinese health study. Int J Hyg Environ Health. 2019;222(2):283-90 Available from: https://doi.org/10.1016/j.jiheh. 2018.12.001.

14. Dalton AM, Jones AP, Sharp SJ, Cooper AJM, Griffin S, Wareham NJ. Residential neighbourhood greenspace is associated with reduced risk of incident diabetes in older people: a prospective cohort study. BMC Public Health. 2016;16(1):1-10 Available from: https://doi.org/10.1186/ s12889-016-3833-z.

15. Lee JJ, Hwang SJ, Mutalik K, Corey D, Joyce R, Block JP, et al. Association of built environment characteristics with adiposity and glycaemic measures. Obes Sci Pract. 2017;3(3):333-41.

16. Tsai HJ, Li CY, Pan WC, Yao TC, Su HJ, Da WC, et al. The effect of surrounding greenness on type 2 diabetes mellitus: a nationwide populationbased cohort in Taiwan. Int J Environ Res Public Health. 2021;18(1):1-11.

17. Bild DE, Bluemke DA, Burke GL, Detrano R, Diez Roux AV, Folsom AR, et al. Multi-ethnic study of atherosclerosis: objectives and design. Am J Epidemiol. 2002;156(9):871-81.

18. Diagnosis and Classification of Diabetes Mellitus. Diabetes Care. 2013, 2014;37(Supplement_1):S81-90. https://doi.org/10.2337/dc14-S081.

19. Klompmaker JO, Hoek G, Bloemsma LD, Gehring U, Strak M, Wijga AH, et al. Green space definition affects associations of green space with overweight and physical activity. Environ Res. 2018;160(May 2017):53140 Available from: https://doi.org/10.1016/j.envres.2017.10.027. 
20. Labib SM, Lindley S, Huck JJ. Spatial dimensions of the influence of urban green-blue spaces on human health: A systematic review. Environ Res. 2020;180(May 2019):108869 Available from: https://doi.org/10.1016/j. envres.2019.108869.

21. Reid CE, Kubzansky LD, Li J, Shmool JL, Clougherty JE. It's not easy assessing greenness: a comparison of NDVI datasets and neighborhood types and their associations with self-rated health in new York City. Heal Place. 2018;54(February):92-101 Available from: https://doi.org/10.1016/j.healt hplace.2018.09.005.

22. Carroll ML, DiMiceli C, Sohlberg R, Townshend J. 250m MODIS normalized difference vegetation index. College Park: Univeristy of Maryland; 2004.

23. Jennings $V$, Bamkole $O$. The relationship between social cohesion and urban green space: an avenue for health promotion. Int J Environ Res Public Health. 2019;16:452. https://doi.org/10.3390/ijerph16030452.

24. Shanahan DF, Bush R, Gaston KJ, Lin BB, Dean J, Barber E, et al. Health benefits from nature experiences depend on dose. Sci Rep. 2016;6(June):1-10 Available from: https://doi.org/10.1038/srep28551.

25. Markevych I, Schoierer J, Hartig T, Chudnovsky A, Hystad P, Dzhambov AM, et al. Exploring pathways linking greenspace to health: theoretical and methodological guidance. Environ Res. 2017;158(February):301-17 Available from: https://doi.org/10.1016/j.envres.2017.06.028.

26. Lee ACK, Maheswaran R. The health benefits of urban green spaces: a review of the evidence. J Public Health (Bangkok). 2011;33(2):212-22.

27. Jones A, Hillsdon M, Coombes E. Greenspace access, use, and physical activity: understanding the effects of area deprivation. Prev Med (Baltim). 2009;49(6):500-5.

28. Cronin-de-Chavez A, Islam S, McEachan RRC. Not a level playing field: a qualitative study exploring structural, community and individual determinants of greenspace use amongst low-income multi-ethnic families. Heal Place. 2019;56(November 2018):118-26 Available from: https://doi.org/ 10.1016/j.healthplace.2019.01.018.

29. U.S. Census Bureau. 2005-2009 American Community Survey--United States [Internet] 2011. Available from: http://www.census.gov/acs/www/ data_documentation/2009_release/

30. U.S. Census Bureau. Census 2000 summary file 1 \& summary file 3 [internet]. Census 2000 Gateway 2001. Available from: https://www.census. gov/main/www/cen2000.html

31. U.S. Census Bureau. 2007-2011 American Community Survey--United States [Internet]. 2013. Available from: https://www.census.gov/newsr oom/releases/archives/news_conferences/20121203_acs5yr.html

32. Moore K, Diez Roux AV, Auchincloss A, Evenson KR, Kaufman J, Mujahid $\mathrm{M}$, et al. Home and work environments in relation to BMI. J Epidemiol Community Health. 2013;67(10):846-53.

33. Mujahid MS, Diez Roux AV, Morenoff JD, Raghunathan T. Assessing the measurement properties of neighborhood scales: from psychometrics to ecometrics. Am J Epidemiol. 2007;165(8):858-67.

34. Troxel W, Matthews K, Bromberger J, Sutton-Tyrrell K. Chronic stress burden, discrimination, and subclinical carotid artery disease in African American and Caucasian women. Health Psychol. 2003;22(3):300-9.

35. Christine PJ. Longitudinal associations between neighborhood physical and social environments and incident type 2 diabetes mellitus. Physiol Behav. 2016;176(1):100-6.

36. Joseph JJ, Echouffo-Tcheugui JB, Golden SH, Chen H, Jenny NS, Carnethon MR, et al. Physical activity, sedentary behaviors and the incidence of type 2 diabetes mellitus : the Multi-Ethnic Study of Atherosclerosis (MESA) Physical activity, sedentary behaviors and the incidence of type 2 diabetes mellitus : the Multi-Ethnic Study of At. BMJ Open Diabetes Res Care. 2016;4(1):e000185.

37. De KC, Tonne C, Nieuwenhuijsen MJ, Singh- A, Sunyer J, Dadvand P, et al. Long-term exposure to greenspace and metabolic syndrome: a Whitehall II study. Environ Pollut. 2019;255(Pt 2):1-20.

38. James P, Banay RF, Hart JE, Laden F. A review of the health benefits of greenness. Curr Epidemiol Reports. 2015;2(2):131-42.

39. Fong $\mathrm{K}$, Hart JE, James $\mathrm{P}$, Medical $\mathrm{H}$, Pilgrim $\mathrm{H}$, Care H. A review of epidemiologic studies on greenness and health: Updated Literature Through 2017. Curr Environ Health Rep. 2018;5(1):77-87. https://doi.org/10.1007/ s40572-018-0179-y.

40. Bilal U, Hill-briggs F, Sánchez-perruca L, Del C-g I, Franco M. Association of neighbourhood socioeconomic status and diabetes burden using electronic health records in Madrid ( Spain ): the HeartHealthyHoods study; 2018.
41. Lamb KE, Thornton LE, King TL, Ball K, White SR, Bentley R, et al. Methods for accounting for neighbourhood self-selection in physical activity and dietary behaviour research: a systematic review. Int J Behav Nutr Phys Act. 2020;17(1):1-22.

42. Li J, Auchincloss AH, Rodriguez DA, Moore KA, Diez Roux AV, Sánchez BN. Determinants of residential preferences related to built and social environments and concordance between neighborhood characteristics and preferences. J Urban Heal. 2020;97(1):62-77.

43. Lin S-H, Young JG, Logan R, VanderWeele TJ. Mediation analysis for a survival outcome with time-varying exposures, mediators, and confounders. Stat Med. 2017;36(26):4153-66.

\section{Publisher's Note}

Springer Nature remains neutral with regard to jurisdictional claims in published maps and institutional affiliations.

Ready to submit your research? Choose BMC and benefit from:

- fast, convenient online submission

- thorough peer review by experienced researchers in your field

- rapid publication on acceptance

- support for research data, including large and complex data types

- gold Open Access which fosters wider collaboration and increased citations

- maximum visibility for your research: over 100M website views per year

At BMC, research is always in progress.

Learn more biomedcentral.com/submissions 